\title{
Temperature during egg formation and the effect of climate warming on egg size in a small songbird
}

\author{
Jaime Potti* \\ Estación Biológica de Doñana - CSIC, Department of Evolutionary Ecology, Pabellón del Perú, Av. M ${ }^{a}$ Luisa s/n, 41013 Sevilla, Spain
}

Keywords:

Breeding phenology

Female condition

Ficedula hypoleuca

Pied flycatcher

\author{
a b s t r a c t
}

The predicted effects of recent climate warming on egg size in birds are controversial, as only two long-term studies have been reported, with contrasting results. Long-term data on egg size variation are analyzed in relation to ambient temperatures in a southern European population of pied flycatchers where breeding phenology has not matched the spring advancement in the last decades. Cross-sectional, population analyses indicated that egg breadth, but not egg length, has decreased significantly along the 16-year period, leading to marginally non-significant decreases in egg volume. Longitudinal, individual analyses revealed that despite females consistently laying larger eggs when they experienced warmer temperatures during the prelaying and laying periods, there was an overall negative response - i.e. decreasing egg volume and breadth with increasing spring (May) average temperatures - across individuals. This trend is hypothesised to be caused by the mismatched breeding phenology, in relation to climate warming, of this population. Except in the unlikely cases of populations capable of perfectly synchronising their phenology to changes in their environment, maladjustments are likely for traits such as egg size, which depend strongly on female condition. Slight changes or absence thereof in breeding dates may be followed by mismatched dates, in terms of food abundance, for optimal egg formation, which would be reflected in smaller average egg size, contrary to early predictions on the effects of climate warming on bird egg size.

\section{Introduction}

Evidence has accumulated in the last few years that recent climate warming has affected the breeding date of some bird populations in North America and Europe, to variable degrees depending on species and geographical location (Parmesan and Yohe, 2003; Visser et al., 2003; Both et al., 2004). For logistic reasons, there are fewer studies examining effects of climate warming on traits other than breeding date or clutch size (Sanz, 2002), with the notable exceptions of the studies by Järvinen (1987) and Tryjanowski et al. (2004). Both these authors examined long-term patterns of population variation in egg size aiming to reveal correlations with increasingly warmer ambient temperatures in their study areas. Järvinen (1987) found an increase of average egg size with warmer temperatures in northern Finnish populations of pied flycatchers (Ficedula hypoleuca), while Tryjanowski et al. (2004) found exactly the opposite trend in a red-backed shrike (Lanius collurio) population in Poland.

* Tel.: p34 95423 2340x174; fax: p34 954621125.

E-mail address: jpotti@ebd.csic.es

1146-609X/\$ - see front matter a 2008 Elsevier Masson SAS. All rights reserved. doi:10.1016/j.actao.2008.02.003 
Jarvinen (1987) predicted, on the basis of the positive relationship between ambient temperatures and average egg sizes he observed in a 19-year study, that climate warming could play a positive effect on some traits such as egg size, 'beneficial' in the sense that a large egg size usually enhances hatching success (Potti and Merino, 1996; Saino et al., 2004). At least in some species larger eggs also result in larger hatchlings, increased growth and development and, ultimately, increased fledging success (Williams, 1994). The pioneer study of Järvinen (1987), based on a cross-sectional population analysis, may nowadays be criticized for a lack of control for other factors in a design that, furthermore, has very low statistical power (Przybylo et al., 2000). Further, as noted by Tryjanowski et al. (2004), predictions about the consequences of climate warming on egg size are not straightforward. This is because egg size is only one of the traits that may be affected by climate warming (Both and Visser, 2005), among many others that may be phenotypically and genetically correlated with it (e.g. individual size or condition; see Potti, 1993; Smith et al., 1993; Williams, 1994; Christians, 2002; Yom-Tov, 2001; Lifjeld et al., 2005). Furthermore, adjustment of egg size to changes in environmental phenology, if any, will depend to a certain degree on a previous adjustment of bird phenology to the phenology of the changing environment. There is evidence that some bird populations, including that studied here, have not substantially advanced their breeding dates in spite of their habitats having experienced increasingly earlier onsets of the springs in the last few decades. In some populations, this has led to mismatches between the timing of peak food supply and nestling demands (Sanz et al., 2003; Both et al., 2004, 2006). Changes in egg size could be further proof of such a mismatch, given its usually strong relationship with female mass or indices of body condition (Christians, 2002).

Here I examine long-term trends of egg size in a population of pied flycatchers studied during 16 years to document patterns of its temporal variation and relationships to ambient temperatures. First, I use detailed data on ambient temperatures experienced by individual females during their laying and immediate prelaying periods, to test whether the variation in egg size in this population has any component attributable to an environmental factor that, for an insectivorous bird, is a strong indicator of resource conditions at the start of breeding. Previous work has centred on genetic and maternal effects on egg size (Potti, 1993, 1999a), on maternal effects due to egg size (Potti and Merino, 1994), and on the consequences of egg size on hatching success (Potti and Merino, 1996). Some purely environmental influences on egg size in this population have also previously been suggested (Potti, 2007), for instance larger eggs were laid in nestboxes of high quality (as measured by rates of nestbox occupancy; Potti, 1993). Increased temperatures experienced by females during laying have been reported to affect positively the egg size of pied flycatchers and other insectivorous passerines (Ojanen et al., 1981; Nager and Zandt, 1994; Perrins, 1996; Stevenson and Bryant, 2000; Hargitai et al., 2005). However, there has been little previous long-term work - as opposed to short-term or experimental studies, which abound (Williams, 1994; Christians, 2002) - on the influence of ambient temperature on the egg size of any altricial bird species that has simultaneously controlled for a set of important factors that may independently affect egg size, such as study year (Hargitai et al., 2005), female mass (Järvinen, 1991; Potti, 1993) or the already mentioned identity effects. Using general linear models (GLM), I test simultaneously for the importance of effects related to ambient temperatures on the egg volume produced by female pied flycatchers, once these relevant influences are taken into account. The longitudinal analysis also examines whether the increased spring warming across the study period, as expressed by decadal variation in average May temperatures (Sanz et al., 2003) has affected egg size. Finally, I explore the fitness consequences of egg size variation and place it in the wider context of long-term breeding patterns by examining its relationship with hatching success and recruitment to the breeding population.

\section{Methods}

I studied the egg size of pied flycatchers breeding in nest boxes in a deciduous (Quercus pyrenaica) forest in La Hiruela, central Spain, during 16 years (1987-2006, with no egg data for the years 1996, 2002 and 2003). All eggs in clutches (range 3-8) were measured during around the sixth day of incubation with callipers to the nearest $0.1 \mathrm{~mm}$ for maximum length (L) and breadth (B) and a mean egg volume $\left(\mathrm{V}\right.$, in $\left.\mathrm{cm}^{3}\right)$ was calculated for each clutch using Hoyt's (1979) formula $\left(\mathrm{V}^{1 / 4} 0.51 \mathrm{LB}^{2}\right)$. The greatest component of variation in egg volume occurs between females, while within-clutch and yearly variation are small (Potti, 1993, 1999a). Egg volume is highly consistent both within clutches and across years and does not vary across the range of female ages (Potti, 1993). Therefore, unique values of egg volumes were obtained by averaging egg volume within a clutch and then across all clutches of individual females.

Females were captured either on the sixth to tenth day of incubation or while feeding nestlings 8-13 days old by means of a trap put within their nest. They were measured for tarsus length (to the nearest $0.05 \mathrm{~mm}$; Potti and Merino, 1994) and weighed with a spring balance (to the nearest $0.1 \mathrm{~g}$ ). In almost all years, masses recorded in the field significantly increased linearly with the time of capture during the day (Potti and Merino, 1995). To adjust for these differences, I transformed field masses to those predicted at noon by using the slopes of yearly linear regressions of mass on time of capture (Potti and Merino, 1995). Female masses recorded during the incubation and the nestling periods differ significantly, precluding lumping of both. As data were limited in many years to only one of those masses, correlations for both are presented in the cross-sectional analysis. For the longitudinal analyses, I used the mass recorded at incubation, for which sample sizes were larger.

For the analyses concerning egg size and temperature during the period of egg formation I computed a 'temperatureduring-prelaying-and-laying' index for each female tailored as a function of her breeding date and clutch size, using the mean daily temperatures experienced by individual females during part of those periods (e.g. Hargitai et al., 2005). To capture most of the temperature range experienced by each female during the period of egg formation in the oviduct (King, 1973), I used the mean of average daily temperatures 
from 4 days before the laying of the first egg up to and including the day before clutch completion (e.g., for a clutch of 6 eggs whose first egg was laid on 15 May, the mean of average daily temperatures from 11 May to 19 May), assuming one egg is laid each day (Lundberg and Alatalo, 1992; Potti, 1993; Potti and Merino, 1996). Meteorological data were supplied by the station of El Vado (Guadalajara), about $17 \mathrm{~km}$ from the study area. In the analysis of temporal trends in egg size only average temperatures in May are analyzed, after checking that trends earlier reported for the period 1980-2001 (Sanz et al., 2003) held until the year 2006. The extended data set showed that temperature in May has indeed significantly increased from 1980 to 2006 (Fig. 1), while neither temperature in April or June nor precipitation in May, April or June have changed in any significant way (Sanz et al., 2003; J. Potti, unpublished data).

To examine sources of environmental variation in egg size while controlling for yearly and individual variation I used restricted maximum likelihood, linear mixed models (GLIMs) as implemented in SAS 9.1 (SAS Institute Inc., 2004). Including female identity as a random factor in the models allows for the presumably operating long-term effects of environmental, genetic and maternal origin on egg size previously suggested for this population (Potti, 1993, 1999a). In these models restricted to females with repeated records of egg size, year and female identity were entered as random factors. Female mass was entered as a fixed covariate on the basis of previous published evidence on its importance in determining an individual's egg size (Potti, 1993, 1999a). Finally, the temperature the female experienced while laying eggs and the yearly means of average May temperatures were also entered as fixed covariates (e.g. Przybylo et al., 2000). Both temperature indices were not strongly correlated ( $\left.\mathrm{r} 1 / 40.47, \mathrm{n}^{1 / 4} 16, \mathrm{P}^{1 / 4} 0.07\right)$ and neither was there a significant time trend in the temperatures experienced by females in their prelaying and laying periods ( $\mathrm{r} 1 / 40.43$, n $1 / 416, \mathrm{P}^{1 / 4} 0.09$ ).

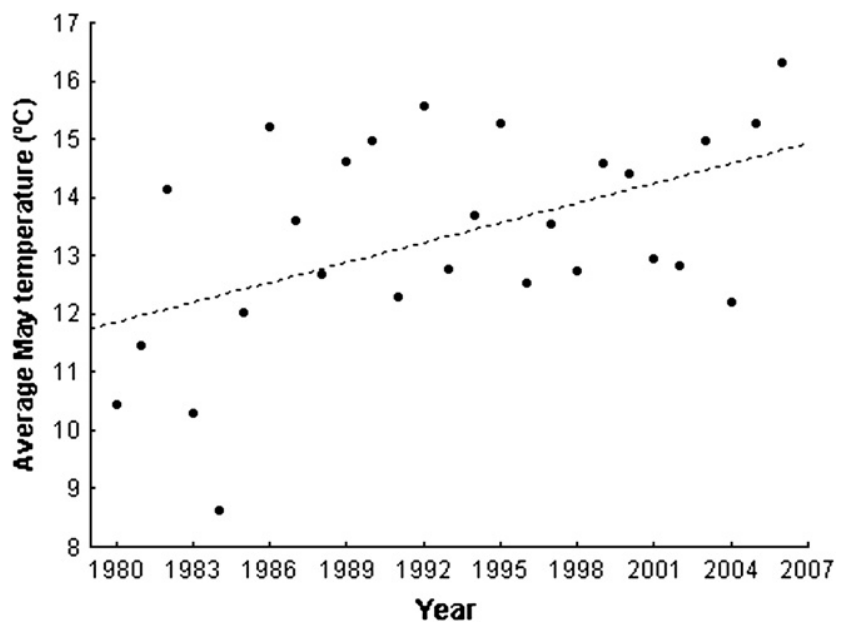

Fig. 1 - Increase in the average temperature in May from 1980 to 2006 near the study area of pied flycatchers in La Hiruela (Madrid). Data supplied by meteorological station of El Vado. The least-squares regression line (average May temperature [ L212.9 D 0.11 8C 3 year) is shown.
To gain insight on the significance of egg size variation, I related the nest's average egg volume to breeding success, in turn decomposed into an (arcsine-transformed) hatching success and the log-transformed numbers of recruits from that nest in the following years. Analyses of recruitment were restricted to the period 1988-2002, when field work was more extensive, making recruitment rates comparable among years. I used GLM models with year as a random factor and egg volume as a predictor variable. Hatching date was also included as a covariate to control for its influence in determining the likelihood of offspring recruitment (Potti et al., 2002).

Repeatabilities were computed by means of the intra-class (within-individual) correlation (r), using variance components from one-way ANOVAs with an individual's identity as factor (Lessells and Boag, 1987). Residuals were tested for normality to meet the assumptions of ANOVAs, which were met in all cases ( $\mathrm{P}>0.20$, Kolmogorov-Smirnov tests). Models were run in SAS 9.1 (SAS Institute Inc., 2004) and additional statistics were computed in Statistica 6.1 (StatSoft, 2002). Sample sizes differ because not all data were taken for all nests in all years.

\section{Results}

Analyses are based on 1244 clutches containing 6350 eggs. Means (SD) of clutch averages were 17.41 (0.66) $\mathrm{mm}$ for egg length, $13.26(0.35) \mathrm{mm}$ for egg breadth and $1.56(0.12) \mathrm{cm}^{3}$ for egg volume. The egg size of female pied flycatchers is rather consistent within females (Potti, 1993, 2007), the updated estimates of repeatability being 0.72 for egg length $\left(F_{294,444} 1 / 47.43\right.$, $\mathrm{P}<0.0001)$, 0.60 for egg breadth $\left(\mathrm{F}_{294,444} 1 / 44.69, \mathrm{P}<0.0001\right)$, and 0.62 for egg volume $\left(\mathrm{F}_{294,444} 1 / 45.16, \mathrm{P}<0.0001\right)$. Given the significant consistency of egg volume and dimensions and the large numbers of repeated records for individual females (n $1 / 4295$ females with data from two or more years), it is clear that female identity should be controlled for in longitudinal analyses. The temperatures experienced by laying females across their breeding attempts were not repeatable $\left(\mathrm{F}_{319,576} 1 / 40.90, \mathrm{P}^{1 / 4} 40.85\right)$, meaning that individual females laid their eggs under a highly variable range of mean ambient temperatures.

\subsection{Cross-sectional analysis}

During the period 1980-2006, average temperatures in May have increased significantly ( $\mathrm{r} 1 / 40.50, \mathrm{n} 1 / 427$, b $1 / 40.11^{\circ} \mathrm{C} /$ year, $\mathrm{P} 1 / 4$ 0.008; Fig. 1), with temperatures in 2006 almost doubling those in the year (1984) the study of pied flycatchers began. During part of this period, average egg dimensions have tended to decrease, significantly so for egg breadth (b $1 / 4-0.01 \mathrm{~mm}$ /year, SE $1 / 40.004, \mathrm{n} 1 / 416, \mathrm{P} \frac{1}{4} 40.03$; Fig. 2) and not significantly for egg length (b $1 / 4-0.002 \mathrm{~mm} /$ year, $\mathrm{SE} 1 / 40.005, \mathrm{n}^{1 / 4} 16, \mathrm{P} 1 / 40.69$; Fig. 2), resulting in a marginally non-significant decrease in average egg volume (b $1 / 4-0.03 \mathrm{~cm}^{3} /$ year, SE $1 / 40.01, \quad \mathrm{n}^{1 / 4} 16, \quad \mathrm{P} \frac{1}{4} 40.08$ ). Although mean female size (tarsus length) did not change across the study period (b $1 / 4-0.004 \mathrm{~mm} /$ year, $\mathrm{SE} 1 / 40.005, \quad \mathrm{P} 1 / 40.44$, $\mathrm{n} 1 / 416$ ), the mass of females has tended to decrease, in a non-significant way for that recorded during the incubation period (b $1 / 4-0.049$ g/year, SE $1 / 40.029, n^{1 / 4} 11, P^{1 / 4} 0.13$; Fig. 3) 

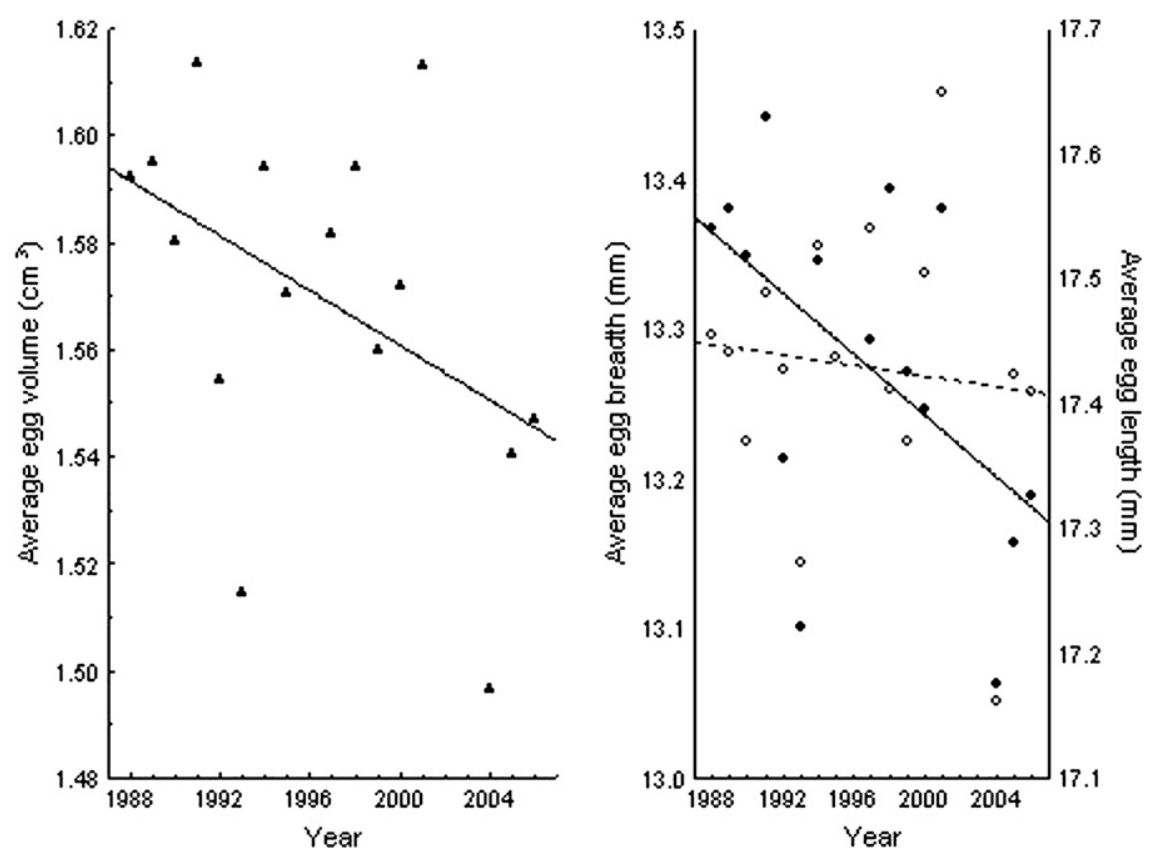

Fig. 2 - Average egg length (open circles, dotted line) and breadth (closed symbols, continuous line) and egg volume from 1988 to 2006. Lines are from linear regressions. Average egg breadth has decreased significantly while declines in average egg length and egg volume are not significant.

and significantly for female mass sampled at the nestling stage (b $1 / 4-0.042$ g/year, SE $1 / 40.012, \mathrm{P}^{1 / 4} 40.005, \mathrm{n} \frac{1}{4} 13$; Fig. 3).

\subsection{Longitudinal analyses}

The linear mixed models revealed significant positive influences of temperatures experienced by females during the prelaying and laying times, so that clutches contained eggs of a larger volume when they were laid at warmer average temperatures (Table 1). In addition, eggs were smaller with increasing average temperatures in May and the analysis also confirmed the significant influence of female mass on egg

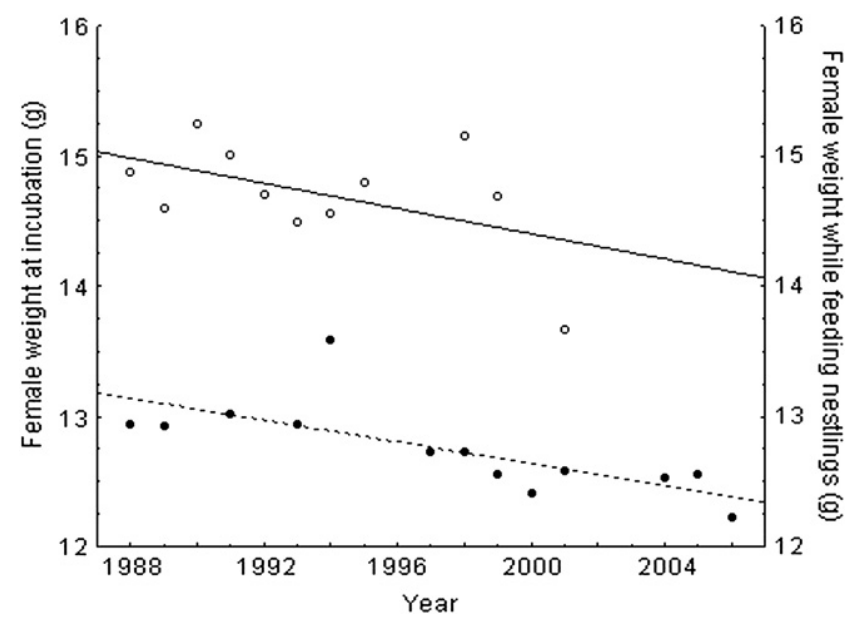

Fig. 3 - Average female mass at incubation (open circles, continuous line) and while feeding nestlings (closed symbols, dashed line) from 1987 to 2006. size (Table 1). As in the cross-sectional analyses, individual decreases in egg volume were due to changes in egg breadth, not in egg length (Table 1).

\subsection{Consequences of variation in egg size}

Females laying larger eggs also enjoyed higher hatching success in their nests (GLM with year as a random factor and egg volume and hatching dates as covariates: year effect $\left(F_{15,832} 1 / 41.95, P 1 / 40.016\right.$; effect of hatching date: $F_{1,832} 1 / 44.07$, $\mathrm{P} 1 / 40.044$; effect of egg volume; $F_{1,832} 1 / 46.93$, $P$ 1/4 0.0086). In turn, although the influence of egg size on recruitment vanished after hatching, an increased hatching success resulted in more offspring recruited to the breeding population (GLM of number of recruits with year as a random factor and egg volume, hatching date and hatching success as fixed covariates; effect of year: $F_{11,522} 1 / 45.75, P<0.0001$; egg size effect: $F_{1,522} 1 / 40.63, P_{1}^{1 / 4} 0.423$; effect of hatching date: $F_{1,522} 1 / 437.48$, $\mathrm{P}<0.0001$; effect of hatching success: $\mathrm{F}_{1,522} \frac{1 / 4}{4} 6.502, \mathrm{P}^{1 / 4} \mathrm{0.001}$ ).

\section{Discussion}

I have shown in this study that the egg size of pied flycatchers has tended to decrease across a 16-year period included within a larger one where climate warming, evidenced in large increases in the average temperatures in May, has negatively affected the reproductive success of the population (Sanz et al., 2003). We attributed this to a mismatch between the advancement of spring (as shown by average May temperatures and the normalized difference vegetation index (NDVI) reflecting tree photosynthetic activity (Myneni et al., 1995; 
Table 1 - Results of type III linear mixed models explaining variation in the egg volume of pied flycatchers

\begin{tabular}{lrrrrr} 
Effect & d.f. & F & P & Estimate & SE \\
\hline Egg volume & & & & & \\
Female mass & 1,198 & 27.84 & $<0.0001$ & 0.030 & 0.006 \\
Laying temperature & 1,198 & 28.94 & $<0.0001$ & 0.014 & 0.003 \\
May temperature & 1,198 & 4.12 & 0.0437 & -0.019 & 0.009 \\
Egg length & & & & & \\
Female mass & 1,198 & 10.67 & 0.0013 & 0.106 & 0.032 \\
Laying temperature & 1,198 & 8.41 & 0.0042 & 0.029 & 0.012 \\
May temperature & 1,198 & 1.75 & 0.1879 & & \\
Egg breadth & & & & & \\
Female mass & 1,198 & 26.29 & 0.0001 & 0.085 & 0.018 \\
Laying temperature & 1,198 & 30.43 & $<0.0001$ & 0.041 & 0.008 \\
May temperature & 1,198 & 4.08 & 0.0448 & -0.056 & 0.027 \\
\hline
\end{tabular}

Year and female identity were entered as random factors and female mass during the incubation stage, average temperature experienced by females during egg laying and average temperature in May were entered as fixed effects. Shown are tests of fixed effects and estimators and their standard errors for significant effects.

Pettorelli et al., 2005) and the average breeding date of the population, that has not 'caught up' to the earlier development of plant productivity in the area (Sanz et al., 2003). Further, that mismatch was reflected by an increasingly deteriorated body condition in both adults and nestlings. The present study adds further evidence that another potentially important fitness component, average egg size, has decreased across the study period in one of those pied flycatcher populations studied on a long-term basis. However, the nature of the decrease in breeding success reported in our earlier work (Sanz et al., 2003) was not attributable to a deteriorated hatching success but to increases in fledgling mortality. The present study confirms that reduced egg size is not responsible for a decreased breeding success, but may instead be reflecting an overall deterioration of breeding conditions that impinges far beyond the effects that egg size may initially have on variation in hatching success.

The longitudinal analyses revealed, first, that the overall response of female pied flycatchers to increases in ambient temperature during egg laying is to increase the size of eggs they lay, as also seen elsewhere (Ojanen et al., 1981; Saino et al., 2004; Hargitai et al., 2005). This has occurred within the context of an increasingly diminishing average female mass, and thus a presumably worsened overall condition, across the study period (Sanz et al., 2003; this study), pointing to the importance of investing in egg size under probably suboptimal conditions. Though I lack data on the availability and abundance of insects in the area, increases in ambient temperature in fresh, montane environments are no doubt accompanied by increases in insect abundance, diversity and activity, enhancing the availability of prey for insectivorous pied flycatchers, and likely translating into the egg-temperature relationship found here. As laying large eggs at low temperatures pushes the females' energetic demands beyond the level at which fitness costs become evident (Stevenson and Bryant, 2000), the correlation between temperature and egg size indicates that females forming eggs probably manage variation in ambient temperatures during this crucial period by allocating more of the resources they gain to their eggs in periods of warmer temperatures and higher prey abundances (Stevenson and Bryant, 2000; Ward and Bryant, 2006). However, temperature during egg formation usually explains a low percentage of the variation in egg size in most studies (Christians, 2002) and my study is no exception, as the variance in egg size explained (the $\mathrm{R}^{2}$ from univariate linear regressions) by ambient temperature during laying time ranged from 0 to $48 \%$ depending on year, with a mean (SD) of $7.0 \%$ (13.6).

In spite of increasingly warmer temperatures in May during the last two and a half decades, which would make predictable larger eggs under Järvinen's (1987) view, and also under the view of decreased thermostatic requirements for females or temperature-dependent food availability (Stevenson and Bryant, 2000) the opposite trend was found. Furthermore, overall decreases in egg size were both concurrent with the increased egg size with warmer daily temperatures during laying and independent of an also increasingly worsening female condition during this period (Sanz et al., 2003). The apparent puzzle, i.e. increasingly warmer temperatures in May being associated to smaller egg sizes despite females laying larger eggs with warmer temperatures during their prelaying and laying periods, is resolved by taking into account that both temperature indices reflect different thermal factors affecting females. While temperatures in the prelaying and laying stages reflect those of specific time intervals experienced by individual females, (average) May temperatures are experienced by almost all females in the population (mean (SD) laying date is 21 (6.9) May (n 1/4 1079), with females arriving on average about 10-12 May (Potti, 1999b) and almost never before 20 April; J. Potti, unpublished data). Thus, the increases in May temperatures have affected almost all females in a similar way, which points to phenotypic plasticity at the individual level. This is also suggested by the similarity in patterns in at least one of the egg dimensions and overall egg size found at both the population (cross-sectional) and individual (longitudinal) levels (see Przybylo et al., 2000). Increasingly smaller eggs with increasingly warmer springs may be reflecting the fact that females are breeding later than optimal under a climate warming scenario, as previously noted for this population (Sanz et al., 2003; Both et al., 2004). As Przybylo et al. (2000) remark, my results also point to the low power of cross-sectional analyses, which collapse widespread individual variation making it hard to detect subtle environmentrelated trends in traits with high levels of genetic and maternal determination, as the one here analyzed (Potti, 1999a; Christians, 2002). Subtle thermal-related trends are in fact clearly shown in this study, as the trends of opposing sign in both temperature indices make manifest.

As stated in Section 1, adjustment of egg size to a changing climate, if present, inevitably depends to some degree on the previous adjustment or mismatch of the birds' phenology to that of their changing environments. Except in the unlikely cases of populations capable of perfectly synchronizing their phenology to changes in their habitats, decreases in traits, such as egg size, dependent to an important degree on female condition (Christians, 2002) seem almost unavoidable. With less food around and out of phase, in relation to environmental phenology, laying females should probably respond by 
decreasing the size of their eggs, as seen here. It may be argued that smaller egg sizes could also be expected for the opposite reasons, i.e. if laying dates match environmental change. This is because, all else being equal, an advanced laying date would be followed by increases in average clutch size in many species (e.g. Hendricks, 2003), including the pied flycatcher (Murphy and Haukioja, 1986; Lundberg and Alatalo, 1992; Both and Visser, 2005). This, at least theoretically, could play a role against increases in egg size due to the possibility of subtle trade-offs between egg and clutch sizes (Nager et al., 2000; Christians, 2002; Figuerola and Green, 2006). However, in addition to the 'stasis' of breeding dates in this population, there has been no indication that clutch size has changed in a significant way across the study period (Sanz et al., 2003), falsifying the idea that decreases in egg size might be linked to increases in clutch size concurrent with slight advancements in breeding date.

What may have been the middle-term consequences of a continued trend for a decreasing egg size in this population of pied flycatchers? As noted by earlier workers (Williams, 1994), and also found in this study, a larger than average egg size enhances hatching success, which is only one of several fitness components. After hatching, other factors go on to impose strong selection pressures and egg size seems to lose importance in the chain of life-history events (fledgling success, survival and recruitment to the breeding population) ultimately leading to an egg's recruitment as a breeder. That is, the importance of egg size for fitness seems to largely vanish after hatching, when the action of other ecological factors, e.g. parental and territory quality, sibling competition, parasitism, predation, etc., may override the initial fitness advantages of laying large eggs (reviewed in Christians, 2002). For instance, although fledgling numbers in my population of pied flycatchers were reduced during the (increasingly warmer) period from 1987 to 2001, hatching success did not change across that interval. This implied that nestling mortality probably caused, as stated above, by a mismatching of breeding dates to the phenological advancement of the spring - was mainly responsible for the observed decrease in breeding success (Sanz et al., 2003). A decreased egg size (this study) may then ultimately represent an added sign of environmental deterioration, but one that pied flycatchers have faced without suffering large fitness losses. Egg size is probably constrained and canalized by genetic and environmental factors, and subjected to selection leaving relatively small margins of individual variation to be detected (Christians, 2002). In a scenario of lasting mismatch to phenological changes, a reduced egg size would not make but reinforce the trend for an increasingly worsened breeding success, by summing up breeding failures at both the egg and the nestling stages. However, in the studied population of pied flycatchers that toll has not yet been paid.

In conclusion, climate warming may generate an intricate pattern of change in reproductive traits of birds (Both and Visser, 2005), depending on the response of bird populations to the changing phenology of their breeding habitats. Slight changes or absence thereof in breeding dates in the face of spring advancements may in turn be followed by mismatched dates, in terms of food abundance, for optimal egg formation. This could be reflected in lower average egg size, contrary to
Järvinen's (1987) prediction and, maybe more importantly for fitness, in lower average egg quality (see Badzinski et al., 2002). More long-term studies are needed to ascertain whether the slight decreases observed herein in pied flycatchers, and by Tryjanowski et al. (2004) in red-backed shrikes, rather than the increase predicted by Järvinen (1987), are the most commonly expected outcomes of recent climate change on egg size in bird populations, and also on the overall acrossspecies importance of egg size variation in relation to individual fitness.

\section{Acknowledgements}

Many thanks to Óscar Frías and David Canal, who helped efficiently over the years. I also thank Luis Prada, from Consejería de Medio Ambiente, Comunidad de Madrid, and Delegación de Medio Ambiente, Junta de Castilla-La Mancha, for working permissions and housing facilities. Birds were trapped under licenses from the ICONA - Spanish Ministry of Agriculture. Paola Laiolo and David Serrano gave much helpful advice. Comments by reviewers helped to improve a first draft. I was supported by projects PAC05-006-1 (to J.A. Dávila), CGL2004-04479/BOS (to J.A. Fargallo) and CGL2006-07481/BOS (to J.C. Senar).

\section{references}

Badzinski, S.S., Davison, C., Leafloor, J.O., Abraham, K.F., 2002. Egg size as a predictor of nutrient composition of eggs and neonates of Canada Geese (Branta canadensis interior) and Lesser Snow Geese (Chen caerulescens caerulescens). Canadian Journal of Zoology 80, 333-341.

Both, C., Visser, M.E., 2005. The effect of climate change on the correlation between avian life-history traits. GlobalChange Biology 11, 1-8.

Both, C., Artemyev, A.V., Blaauw, B., Cowie, R., Dekhuizen, H., Gustafsson, L., Järvinen, A., Kerimov, A., Metcalfe, N., Potti, J., Ravussin, P.A., Sanz, J.J., Silverin, B., Slater, F., Török, J., Winkel, W., Wright, J., Zang, H., Visser, M.E., 2004. Large-scale geographical variation confirms that climate change causes birds to lay earlier. Proceedings of the Royal Society of London B 271, 1657-1662.

Both, C., Bouwhuis, S., Lessells, C.M., Visser, M.E., 2006. Climate change and population declines in a long-distance migratory bird. Nature 441, 81-83.

Christians, J.K., 2002. Avian egg size: variation within species and inflexibility within individuals. Biological Reviews 77, 1-26.

Figuerola, J., Green, A.J., 2006. A comparative study of egg mass and clutch size in the Anseriformes. Journal of Ornithology 147, 57-68.

Hargitai, R., Török, J., Tó th, L., Hegyi, G., Rosivall, B.Z., Szigeti, B., Szö lløsi, E., 2005. Effects of environmental conditions and parental quality on inter- and intraclutch egg-size variation in the collared flycatcher (Ficedula albicollis). Auk 122, 509-522.

Hendricks, P., 2003. Spring snow conditions, laying date, and clutch size in an alpine population of American Pipits. Journal of Field Ornithology 74, 423-429.

Hoyt, D.F., 1979. Practical methods of estimating volume and fresh weight of bird eggs. Auk 96, 73-77.

Järvinen, A., 1987. Global warming and egg size of birds. Ecography 17, 108-110. 
Järvinen, A., 1991. Proximate factors affecting egg volume in subarctic hole-nesting passerines. Ornis Fennica 68, 99-104.

King, J.R., 1973. Energetics of reproduction in birds. In: Farner, D.S. (Ed.), Breeding Biology of Birds. Academic Press, New York, pp. 78-107.

Lessells, C.M., Boag, P.T., 1987. Unrepeatable repeatabilities: a common mistake. Auk 104, 116-121.

Lifjeld, J.T., Johnsen, A., Petitguyot, T., 2005. Egg-size variation in the bluethroat (Luscinia s. svecica): constraints and adaptation. Journal of Ornithology 146, 249-256.

Lundberg, A., Alatalo, R.V., 1992. The Pied Flycatcher. Poyser, London.

Murphy, E.C., Haukioja, E., 1986. Clutch size in nidicolous birds. Current Ornithology 4, 141-210.

Myneni, R.B., Hall, F.G., Sellers, P.J., Marshak, A.L., 1995. The interpretation of spectral vegetation indexes. IEEE

Transactions on Geoscience and Remote Sensing 33, 481-486.

Nager, R.G., Zandt, H.S., 1994. Variation in egg size in Great Tits. Ardea 82, 315-328.

Nager, R.G., Monaghan, P., Houston, D.C., 2000. Within-clutch trade-offs between the number and quality of eggs: experimental manipulations in gulls. Ecology 81, 1339-1350.

Ojanen, M., Orrell, M., Väisänen, R.A., 1981. Egg size variation within passerine clutches: effects of ambient temperature and laying sequence. Ornis Fennica 58, 93-108.

Parmesan, C., Yohe, G., 2003. A globally coherent fingerprint of climate change impacts across natural systems. Nature 421 , 37-42.

Perrins, C.M., 1996. Eggs, egg formation and the timing of breeding. Ibis 138, 2-15.

Pettorelli, N., Vik, J.O., Mysterud, A., Gaillard, J.M., Tucker, C.J., Stenseth, N.C., 2005. Using the satellite-derived NDVI to assess ecological responses to environmental change. Trends in Ecology and Evolution 20, 503-510.

Potti, J., 1993. Environmental, ontogenetic and genetic variation in egg size of pied flycatchers. Canadian Journal of Zoology 71, $1534-1542$.

Potti, J., 1999a. Maternal effects and the pervasive impact of nestling history on egg size in a passerine bird. Evolution 53, 279-285.

Potti, J., 1999b. From mating to laying: genetic and environmental variation in mating dates and prelaying periods of female Pied Flycatchers Ficedula hypoleuca. Annales Zoologici Fennici 36, 187-194.

Potti, J., 2007. Male site-tenacity reveals an environmental contribution to egg size in pied flycatchers Ficedula hypoleuca. Ardeola 54, 117-121.

Potti, J., Merino, S., 1994. Heritability estimates and maternal effects on tarsus length in pied flycatchers. Ficedula hypoleuca. Oecologia 100, 331-338.
Potti, J., Merino, S., 1995. Female mass losses are related to male age and body condition in the Pied Flycatcher Ficedula hypoleuca. Ardeola 42, 173-181.

Potti, J., Merino, S., 1996. Causes of hatching failure in Pied Flycatchers. Condor 98, 328-336.

Potti, J., Dávila, J.A., Tella, J.L., Frías, Ó., Villar, S., 2002. Gender and viability selection on morphology in fledgling pied flycatchers. Molecular Ecology 11, 1317-1326.

Przybylo, R., Sheldon, B.C., Merilä, J., 2000. Climatic effects on breeding and morphology: evidence for phenotypic plasticity. Journal of Animal Ecology 69, 395-403.

Saino, N., Romano, M., Ambrosini, R., Ferrari, R.P., Møller, A.P., 2004. Timing of reproduction and egg quality covary with temperature in the insectivorous Barn Swallow. Hirundo rustica. Functional Ecology 18, 50-57.

Sanz, J.J., 2002. Climate change and breeding parameters of great and blue tits throughout the western Palaearctic. Global Change Biology 8, 409-422.

Sanz, J.J., Potti, J., Moreno, J., Merino, S., Frías, Ó., 2003. Fitness consequences of climate change in a migratory bird breeding in the Mediterranean region. Global Change Biology 9, 1-12.

SAS Institute Inc., 2004. SAS/STAT 9.1 User's Guide. SAS Institute Inc, Cary.

Smith, H.G., Ottosson, U., Ohlsson, T., 1993. Interclutch variation in egg mass among Starlings Sturnus vulgaris reflects female condition. Ornis Scandinavica 24, 311-316.

StatSoft Inc., 1996. Statistica for Windows, Release 5.1 (Computer Program Manual). Tulsa.

Stevenson, I.R., Bryant, D.M., 2000. Climate change and constraints on breeding. Nature 406, 366-367.

Tryjanowski, P., Sparks, T.H., Kuczyński, L., Kuzńiak, S., 2004. Should avian egg size increase as a result of global warming? A case study using the red-backed shrike (Lanius collurio). Journal of Ornithology 145, 264-268.

Visser, M.E., Adriaensen, F., van Balen, J.H., Blondel, J., Dhondt, A. A., van Dongen, S., du Feu, C., Ivankina, E.V., Kerimov, A.B., de Laet, J., Matthysen, E., McCleery, R., Orell, M., Thomson, D.L., 2003. Variable responses to large-scale climate change in European Parus populations. Proceedings of the Royal Society of London B 270, 367-372.

Ward, S., Bryant, D.M., 2006. Barn swallows Hirundo rustica form eggs mainly from current food intake. Journal of Avian Biology 37, 179-189.

Williams, T.D., 1994. Intraspecific variation in egg size and egg composition in birds: effects on offspring fitness. Biological Reviews 68, 35-59.

Yom-Tov, Y., 2001. Global warming and body mass decline in Israeli passerine birds. Proceedings of the Royal Society of London B 268, 947-952. 